\title{
LAYERED CIRCLE PACKINGS
}

\author{
DAVID DENNIS AND G. BROCK WILLIAMS
}

Received 14 January 2005 and in revised form 23 June 2005

Given a bounded sequence of integers $\left\{d_{0}, d_{1}, d_{2}, \ldots\right\}, 6 \leq d_{n} \leq M$, there is an associated abstract triangulation created by building up layers of vertices so that vertices on the $n$th layer have degree $d_{n}$. This triangulation can be realized via a circle packing which fills either the Euclidean or the hyperbolic plane. We give necessary and sufficient conditions to determine the type of the packing given the defining sequence $\left\{d_{n}\right\}$.

\section{Introduction}

Since Thurston's conjecture that maps between circle packings could be used to approximate conformal maps $[7,9]$, properties of circle packings have been intensely studied as discrete analogues of classical conformal geometry. For example, Beardon and Stephenson showed that a triangulation of an open topological disc has a packing which fills either the Euclidean or the hyperbolic plane [1]. This is analogous to the classical uniformization theorem which states that any open topological disc can be mapped conformally onto either the Euclidean or the hyperbolic plane.

Many authors have considered the resulting type problem deciding whether a given triangulation produces a parabolic (filling the Euclidean plane) or hyperbolic packing $[3,4,5,8]$. He and Schramm have given the most complete answer, producing characterizations in terms of discrete extremal length, random walks, and electrical resistance [5]. These conditions can often be difficult to check in practice; thus, we give a more direct characterization for the special case of layered circle packings.

Layered packings are created from a bounded defining sequence $\left\{d_{n}\right\}$ in such a way that all the circles in the $n$th layer are tangent to exactly $d_{n}$ other circles. For infinite layered packings, we formulate a type criterion based solely on the defining sequence $\left\{d_{n}\right\}$.

This extends earlier work of Siders [8], who considered only the case $d_{n}=6$ or 7 . It follows from the length-area lemma of Rodin and Sullivan [7] that a packing is parabolic if the sum of the reciprocals of the number of neighbors in each generation is infinite. Theorem 3.6 implies that condition is both necessary and sufficient for layered packings. 


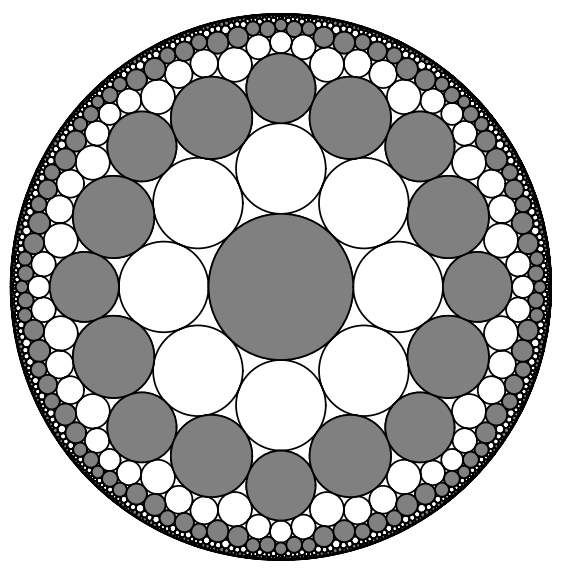

Figure 2.1. A finite portion of the layered circle packing created from the defining sequence $\{8,6,8,6, \ldots\}$. The circles of degree 8 are shaded.

\section{Circle packings}

Consider a collection of nonoverlapping circles in the Euclidean or hyperbolic plane. We can analyze its combinatorial structure by examining its tangency graph, formed by associating each circle to a vertex and connecting by an edge those vertices whose corresponding circles are tangent. Notice that the circles themselves generate a geometric realization of their tangency graph by placing vertices at the centers of the circles they represent and embedding edges as geodesic segments connecting these centers. The region thus covered by the embedded tangency graph is called the carrier.

Definition 2.1. A circle packing is a locally finite configuration of circles whose tangency graph is a triangulation. A packing fills a surface if its carrier covers the entire surface.

As it is common in the literature, we limit our attention here to packings with a global bound on the degree of each vertex, that is, a bound on the number of edges incident at any given vertex. Given a bounded sequence of integers $\left\{d_{0}, d_{1}, d_{2}, \ldots\right\}, 6 \leq d_{n} \leq M$, we can construct a layered triangulation by building up layers of vertices so that the $n$th layer has degree $d_{n}$. Such a triangulation can then be realized by a layered circle packing. Notice that we begin enumerating the layers starting at 0 . Layer number 0 consists of a single central circle $C_{0}$ surrounded by $d_{0}$ other circles, see Figure 2.1 .

Definition 2.2. For $n>0$, the order of a vertex in the $n$th layer is the number of its neighbors in the $(n-1)$ th layer.

Proposition 2.3. If $d_{n} \geq 6$ for all $n$, then the order of every circle in the corresponding layered circle packing is either 1 or 2 .

Proof. Notice that all circles in the first layer are tangent to the same central circle $C_{0}$. Thus all circles in the first layer have order 1. 


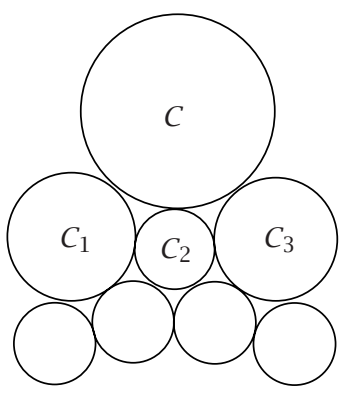

Figure 2.2. The circle $C$ has three consecutive neighbors $C_{1}, C_{2}$, and $C_{3}$ in the previous layer. If $C_{2}$ has order at most 2 , then it can have degree at most 5 .

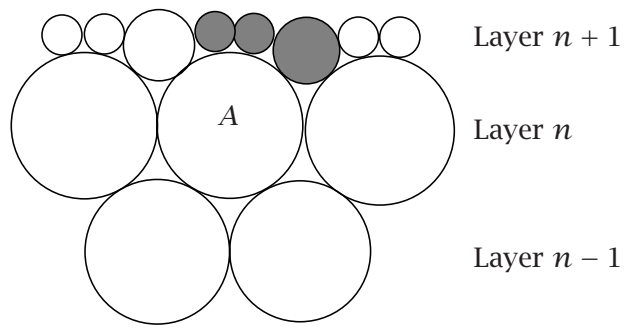

Figure 2.3. The shaded circles are the offspring of circle $A$.

Now assume that all circles in the first $n$ layers have order either 1 or 2 , but some circle $C$ in the $(n+1)$ th layer has order greater than 2 . Then $C$ has at least three consecutive neighbors $C_{1}, C_{2}$, and $C_{3}$ in the $n$th layer. By our assumption, $C_{2}$ has at most 2 neighbors in the $(n-1)$ th layer, see Figure 2.2. In the $n$th layer, $C_{2}$ has exactly 2 neighbors, namely $C_{1}$ and $C_{3}$. But the only circle in the $(n+1)$ th layer tangent to $C_{2}$ is $C$ since both $C_{1}$ and $C_{3}$ are also tangent to $C$. Consequently, $C_{2}$ can have degree at most 5 , contradicting our assumption that $d_{n} \geq 6$.

The offspring of a vertex in the $n$th layer, $n>0$, are its neighbors in the $(n+1)$ th layer listed counter-clockwise from its rightmost neighbor up to, but not including, its leftmost neighbor. The central circle $C_{0}$ is considered to have $d_{0}$ offspring. Thus every circle in the $(n+1)$ th layer is the offspring of exactly one circle in the $n$th layer, see Figure 2.3.

\section{Determining the type}

The discrete uniformization theorem of Beardon and Stephenson [1] states that every infinite simply connected triangulation has a circle packing which fills either the Euclidean plane $\mathbb{C}$ or the hyperbolic plane $\mathbb{D}$ (but not both). In the first case, the triangulation and corresponding packing are designated parabolic, in the second, hyperbolic. Determining which category a given triangulation falls into is referred to as the type problem.

He and Schramm [5] and Doyle and Snell [2] have produced several important reformulations of the type problem. 
Theorem 3.1. If the defining sequence $\left\{d_{n}\right\}$ for a layered circle packing $\mathscr{P}$ is bounded, then the following are equivalent.

(i) The graph $\mathcal{K}$ for $\mathscr{P}$ is parabolic.

(ii) The simple random walk on $\mathscr{K}$ is recurrent.

(iii) The electrical resistance to infinity of $\mathscr{Y}$ is infinite.

The electrical resistance to infinity of a graph is determined by treating $\mathscr{K}$ as an electrical network in which each edge is a wire with unit resistance. Now fix some vertex $v_{0}$ and consider the finite portion $\mathcal{K}_{m}$ of $\mathscr{K}_{\mathcal{K}}$ consisting of all vertices and edges $m$ generations from $v_{0}$. Apply a unit voltage at $v_{0}$, ground out the boundary, and compute the resistance $R_{m}$ from $v_{0}$ to the boundary. The resistance of $\mathscr{K}$ to infinity is

$$
R=\lim _{m \rightarrow \infty} R_{m}
$$

One should imagine an electron as a random walker starting at $v_{0}$ and wandering around the graph. If the resistance to infinity is infinite, the electron cannot "escape," and must return to $v_{0}$. In this case, the circle packing corresponding to $\mathscr{K}$ must be parabolic. Heuristically, there are not enough circles in each generation to allow the electron to get lost.

Conversely, if the electron can "escape" to infinity, there must be many more circles in each generation. This can only happen in the hyperbolic plane, where there is much more room at infinity.

For layered packings, the electrical resistance can be easily described combinatorially.

THEOREM 3.2. The electrical resistance to infinity of the graph $\mathscr{Y}$ of a layered circle packing $\mathscr{P}$ is infinite if and only if the sum

$$
\sum_{n=1}^{\infty} \frac{1}{E_{n}}
$$

is infinite, where $E_{n}$ is the number of edges connecting vertices in the $n$th and $(n-1)$ th layers. Proof. Suppose that $\sum_{n=1}^{\infty}\left(1 / E_{n}\right)=\infty$. Recall that every order-1 vertex $v$ in $\mathscr{K}$ is connected to exactly one vertex $w$ in the previous layer. For each such $v$, add a second parallel edge connecting $v$ and $w$, see Figure 3.1. This new augmented graph $\mathscr{K}^{*}$ will obviously no longer be a triangulation, but the resistance to infinity is still defined and its electrical interpretation is still valid. By Rayleigh's monotonicity law, the resistance to infinity $R^{*}$ of $\mathscr{K}^{*}$ is no greater than the resistance $R$ of $\mathscr{K}[2,6]$. (Heuristically, adding edges makes it easier for electrons to escape to infinity.) Moreover, if $E_{n}^{*}$ is the number of edges in $\mathscr{K}^{*}$ connecting vertices in the $(n-1)$ th and $n$th layers, then

$$
E_{n}<E_{n}^{*}<2 E_{n}
$$

Let $\mathcal{K}_{m}$ and $\mathscr{K}_{m}^{*}$ denote the portions of $\mathscr{K}$ and $\mathscr{K}^{*}$ consisting of the first $m$ layers about the central vertex $v_{0}$. Notice that if $d_{0}$ is the degree of $v_{0}$, then $\mathcal{K}_{m}^{*}$ possesses $d_{0}$-fold rotational symmetry. Since the voltage at each vertex is uniquely determined by the boundary 


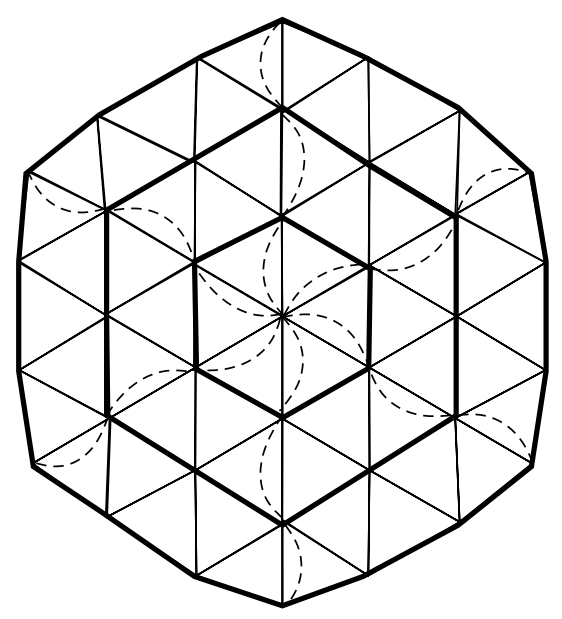

Figure 3.1. Adding edges (dashed) to $\mathscr{K}_{3}$ produces an augmented graph $\mathscr{K}_{3}^{*}$. Vertices on the same layer are indicated by the darker lines.

conditions, the voltage at each of the neighbors of $v_{0}$ must be the same. Thus we can short out this first layer and replace it by a single vertex $v_{1}$ without changing the resistance.

Next, notice that every vertex in the second layer of $\mathscr{K}_{m}^{*}$ is connected; now connected by exactly two edges to $v_{1}$. The new network is $d_{1}$-fold rotationally symmetric, so we can short out the second layer and replace it by a single vertex $v_{2}$. Repeating this process for the remaining layers, we form a series of vertices $v_{0}, v_{1}, \ldots, v_{m}$ with $v_{n-1}$ and $v_{n}$ connected by $E_{n}^{*}$ unit resistors in parallel, $n=1,2, \ldots, m$. The effective resistance between $v_{n-1}$ and $v_{n}$ is then just $1 / E_{n}^{*}$, and we can replace these $E_{n}^{*}$ different resistors with a single resistor of resistance $1 / E_{n}^{*}$, see Figure 3.2.

Thus we can reduce the network $\mathscr{K}_{m}^{*}$ to a series of $m$ resistors having resistances $1 / E_{1}^{*}$, $1 / E_{2}^{*}, \ldots, 1 / E_{m}^{*}$. The total resistance from $v_{0}$ to the boundary must then be

$$
R_{m}^{*}=\sum_{n=1}^{m} \frac{1}{E_{n}^{*}} .
$$

Since adding edges to the triangulation $\mathscr{K}_{m}$ does not increase its resistance $R_{m}$, we have

$$
R_{m} \geq R_{m}^{*}=\sum_{n=1}^{m} \frac{1}{E_{n}^{*}}>\sum_{n=1}^{m} \frac{1}{2 E_{n}} .
$$

Consequently, the resistance of $\mathscr{K}$ to infinity is

$$
R=\lim _{m \rightarrow \infty} R_{m} \geq \sum_{n=1}^{\infty} \frac{1}{2 E_{n}}=\infty
$$

and $\mathscr{K}$ is parabolic. 


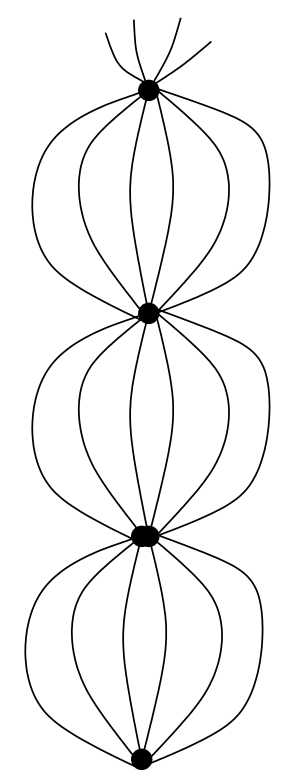

Figure 3.2. After shorting out the vertices on the same layer, the network reduces to a series of $m$ vertices connected by $E_{n}^{*}$ unit resistors in parallel.

Conversely, if $\sum_{n=1}^{\infty}\left(1 / E_{n}\right)<\infty$, then we create a new graph $\mathcal{K}_{m}^{*}$ from $\mathcal{K}_{m}$ by cutting one of the edges, say the leftmost edge, joining each order-2 vertex to the previous layer. Now every vertex in the $n$th layer of $\mathcal{K}_{m}^{*}$ is joined to the previous layer by exactly one edge. If $E_{n}^{*}$ is the number of edges in $\mathcal{K}_{m}^{*}$ connecting vertices in the $(n-1)$ th and $n$th layers, then

$$
\frac{1}{2} E_{n}<E_{n}^{*}<E_{n}
$$

Again we use the symmetry of the entire graph to short out the first layer. Now the resulting graph will be $d_{1}$-fold rotationally symmetric and we can short out the second layer. Proceeding in this way, we compute the resistance from $v_{0}$ to the boundary of $\mathcal{K}_{m}^{*}$ to be

$$
\sum_{n=1}^{m} \frac{1}{E_{n}^{*}}
$$

By Rayleigh's monotonicity law, cutting edges does not decrease resistance $[2,6]$. Thus the resistance of $\mathscr{K}$ to infinity is

$$
R \leq \sum_{n=1}^{\infty} \frac{1}{E_{n}^{*}} \leq \sum_{n=1}^{\infty} \frac{1}{(1 / 2) E_{n}}<\infty,
$$

and $\mathscr{K}$ is hyperbolic. 
Proposition 3.3. Let $V_{0}=0$ and let $V_{n}$ be the number of vertices in the $n$th layer for $n>0$. Then $E_{n}=V_{n}+V_{n-1}$.

Proof. Every order- 1 vertex in the $n$th layer is connected by exactly one edge to some vertex in the previous layer. Similarly, every order- 2 vertex is connected by exactly two edges to some vertex in the previous layer. Thus if $V_{n, 1}$ is the number of order-1 vertices in the $n$th layer and $V_{n, 2}$ is the number of order- 2 vertices in the $n$th layer, then

$$
E_{n}=V_{n, 1}+2 V_{n, 2}
$$

Notice that each order- 2 circle fits in the "gap" between two adjacent circles in the previous layer. Since there are as many of these "gaps" as there are vertices in the previous layer, $V_{n, 2}=V_{n-1}$. Thus

$$
\begin{aligned}
E_{n} & =V_{n, 1}+2 V_{n, 2} \\
& =V_{n}+V_{n, 2} \\
& =V_{n}+V_{n-1} .
\end{aligned}
$$

Lemma 3.4. Every order-1 vertex in the $n$th layer has $d_{n}-4$ offspring, while every order-2 vertex has $d_{n}-5$ offspring.

Proof. The number of offspring corresponding to a vertex $v$ is the number of unique neighbors to that vertex in the next layer, that is, all of the neighbors in the next layer except the leftmost one which we designate as an offspring of $v$ 's left neighbor in the same layer. Hence, we have that the number of offspring, for an order-1 vertex is its degree, $d_{n}$, minus the 3 neighbors that are already in place in the $n$th and $(n-1)$ th layers and also the 1 neighbor in the $(n+1)$ th layer that is shared and designated as the offspring for a different vertex. Consequently, an order-1 vertex must have $\left(d_{n}-4\right)$ offspring. On the other hand, since order-2 vertices have an extra neighbor in the $(n-1)$ th layer, they have only $\left(d_{n}-5\right)$ offspring.

Lemma 3.4 now yields a second-order recurrence relation for $V_{n}$.

LEMma 3.5. For $n>1$, the number of vertices contained in the nth layer satisfies the secondorder recurrence relation

$$
V_{n}=\left(d_{n-1}-4\right) V_{n-1}-V_{n-2}
$$

Proof. To determine $V_{n}$, we must count the offspring of the $(n-1)$ th layer $L_{n-1}$. By Lemma 3.4,

$$
\begin{aligned}
V_{n}= & \left(d_{n-1}-4\right)\left(\text { the number of order-1 vertices in } L_{n-1}\right) \\
& +\left(d_{n-1}-5\right)\left(\text { the number of order- } 2 \text { vertices in } L_{n-1}\right)
\end{aligned}
$$


By Definition 2.2, the number of order-2 vertices in $L_{n-1}$ is $V_{n-2}$. Because there are only order-1 and order- 2 vertices, the number of order- 1 vertices in $L_{n-1}$ is $V_{n-1}-V_{n-2}$. Thus,

$$
\begin{aligned}
V_{n} & =\left(d_{n-1}-4\right)\left(V_{n-1}-V_{n-2}\right)+\left(d_{n-1}-5\right) V_{n-2} \\
& =\left(d_{n-1}-4\right) V_{n-1}+\left[\left(d_{n-1}-5\right)-\left(d_{n-1}-4\right)\right] V_{n-2} \\
& =\left(d_{n-1}-4\right) V_{n-1}-V_{n-2} .
\end{aligned}
$$

Combining Proposition 3.3 and Lemma 3.5, we have that

$$
\begin{aligned}
E_{n} & =V_{n}+V_{n-1} \\
& =\left[\left(d_{n-1}-4\right) V_{n-1}-V_{n-2}\right]+\left[\left(d_{n-2}-4\right) V_{n-2}-V_{n-3}\right] \\
& =\left(d_{n-1}-4\right) V_{n-1}+\left(d_{n-2}-5\right) V_{n-2}-V_{n-3} .
\end{aligned}
$$

Thus, we can compute $\sum\left(1 / E_{n}\right)$ by solving the recurrence equation for $V_{n}$. On the other hand, we can reformulate our type criterion directly in terms of the numbers of vertices.

Theorem 3.6. A layered circle packing with (bounded) defining sequence $\left\{d_{n}\right\}, d_{n} \geq 6$, is hyperbolic if and only if

$$
\sum_{n=1}^{\infty} \frac{1}{V_{n}}<\infty,
$$

where $V_{n}$ is the number of vertices in the nth layer, $n>0$. Moreover, $V_{n}$ is given by the second-order recurrence relation

$$
V_{n}=\left(d_{n-1}-4\right) V_{n-1}-V_{n-2}
$$

with initial conditions $V_{0}=0$ and $V_{1}=d_{0}$.

Proof. Recall that $\left\{d_{n}\right\}$ is bounded, say $d_{n} \leq M$, for all $n$. Since each vertex has at most $M$ neighbors, $E_{n} \leq M V_{n}$.

Moreover, since $E_{n}=V_{n}+V_{n-1}$ by Proposition 3.3,

$$
V_{n} \leq E_{n} \leq M V_{n}
$$

Thus, for layered packings, $\sum\left(1 / E_{n}\right)$ converges or diverges as $\sum\left(1 / V_{n}\right)$ does, and by Theorem 3.2, the convergence of $\sum\left(1 / E_{n}\right)$ is equivalent to the hyperbolicity of the packing.

\section{Examples}

We now consider several examples of layered packings with defining sequences which can be easily analyzed.

One of the first packings to be studied is the "hex packing" in which every circle has degree 6 and is well known to be parabolic $[7,10]$. In this case, $d_{n} \equiv 6$, and solving our 


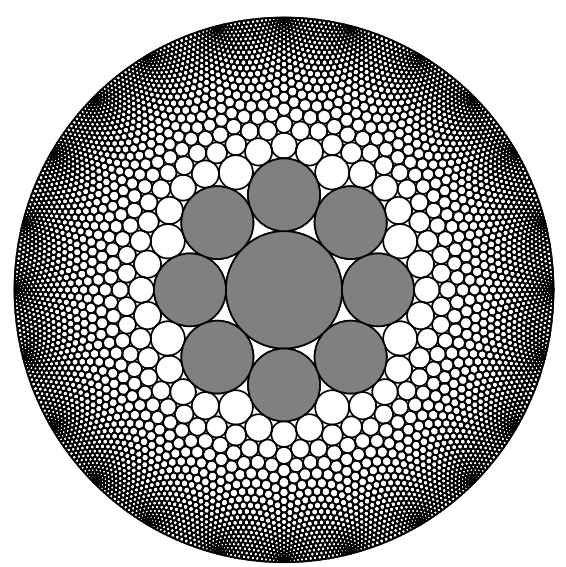

Figure 4.1. A portion of the layered packing generated by the sequence $\{8,8,6,6,6, \ldots\}$. The circles of degree 8 are shaded.

recursion relation above yields

$$
V_{n}=6 n
$$

Thus $\sum_{n=1}^{\infty}\left(1 / V_{n}\right)$ is indeed infinite.

On the other hand, packings of constant degree $d>6$ are known to be hyperbolic $[1,5]$. In this case,

$$
V_{n}=\frac{d\left(\left(d-4+\sqrt{(d-4)^{2}-4}\right)^{n}-\left(d-4-\sqrt{(d-4)^{2}-4}\right)^{n}\right)}{2^{n} \sqrt{(d-4)^{2}-4}}
$$

and $\sum_{n=1}^{\infty}\left(1 / V_{n}\right)$ is finite.

More interesting examples arise by mixing layers of degree 6 with higher-degree layers. For example, the sequence $\{8,8,6,6,6, \ldots\}$ produces parabolic packing with

$$
V_{n}=24 n-16,
$$

for $n>0$, see Figure 4.1 .

\section{Layers of degree five}

Recall that if $d_{n} \geq 6$, for all $n$, then an infinite layered packing always exists. But if $d_{n}=5$ for some $n$, then a packing might exist only for a finite portion of the sequence. For example, the sequence $\{5,5,5\}$ produces a layered packing that folds up into a sphere.

Intuitively, degree-5 layers "want" to produce a spherical packing, degree-6 layers "want" to produce a parabolic packing, and layers of degree 7 or higher "want" to produce a hyperbolic packing. One might naively guess that alternating layers of degree 5 


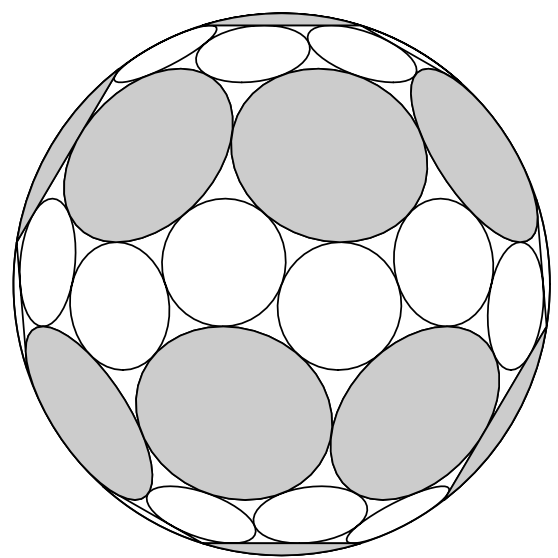

Figure 5.1. The layered circle packing with defining sequence $\{7,5,7,5,7\}$. The circles of degree 7 are shaded. Notice that the combinatorics forces the packing to cover a sphere.

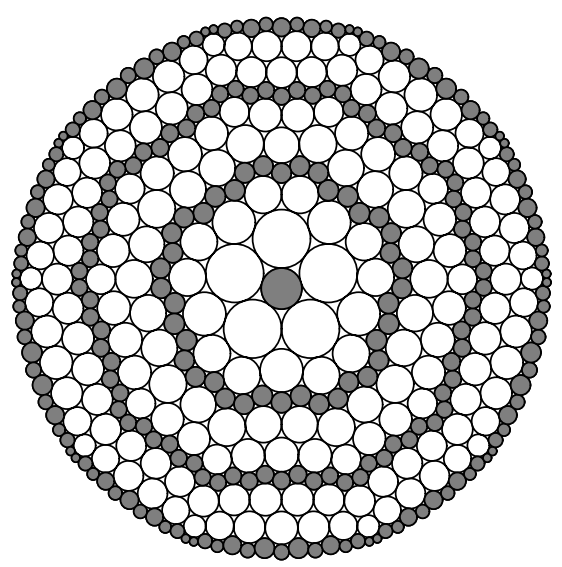

Figure 5.2. A portion of the layered packing generated by the sequence $\{5,7,7,5,7,7, \ldots\}$. The circles of degree 5 are shaded.

and 7 should "average out" to a parabolic 6 , however, this is not the case. The sequences $\{7,5,7,5,7\}$ and $\{5,7,5,7,5\}$ also produce spherical packings, see Figure 5.1 .

On the other hand, the sequence $\{5,7,7,5,7,7, \ldots\}$ does produce a parabolic packing. If $n=3 m+j$, then

$$
V_{n}= \begin{cases}\frac{40}{3} n, & j=0, \\ 5+10(n-m-1), & j=1,2\end{cases}
$$

see Figure 5.2. 


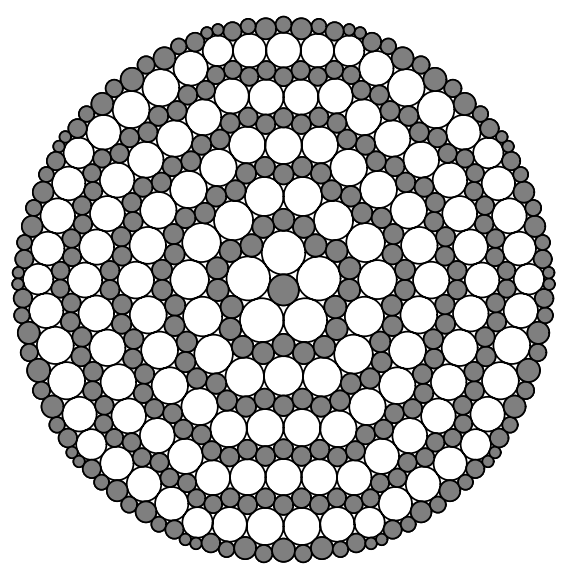

Figure 5.3. A portion of the layered packing generated by the sequence $\{5,8,5,8, \ldots\}$. The circles of degree 5 are shaded.

Similarly, the layered packing for the periodic sequence $\{5,8,5,8, \ldots\}$ is also parabolic, having

$$
\begin{aligned}
V_{2 n} & =20 n, \\
V_{2 n+1} & =10 n+5,
\end{aligned}
$$

see Figure 5.3. A " 5 " thus requires either two " 7 's" or one " 8 " to reach a parabolic equilibrium.

For layered packings with $d_{n}=5$ for some $n$, Proposition 2.3 no longer holds. Thus the recurrence relation for $E_{n}$ must include terms for more than two preceding layers, and consequently is somewhat less useful in practice.

\section{Acknowledgments}

The first author gratefully acknowledges the support of the Texas Tech Honors Research Fellowship Program and Department of Mathematics and Statistics. The second author gratefully acknowledges the support of the Texas Tech Research Enhancement Fund.

\section{References}

[1] A. F. Beardon and K. Stephenson, The uniformization theorem for circle packings, Indiana Univ. Math. J. 39 (1990), no. 4, 1383-1425.

[2] P. G. Doyle and J. L. Snell, Random Walks and Electric Networks, Carus Mathematical Monographs, vol. 22, Mathematical Association of America, Washington, DC, 1984.

[3] T. Dubejko, Random walks on circle packings, Lipa’s Legacy (New York, 1995), Contemp. Math., vol. 211, American Mathematical Society, Rhode Island, 1997, pp. 169-182.

[4] Lecurrent random walks, Liouville's theorem and circle packings, Math. Proc. Cambridge Philos. Soc. 121 (1997), no. 3, 531-546.

[5] Z.-X. He and O. Schramm, Hyperbolic and parabolic packings, Discrete Comput. Geom. 14 (1995), no. 2, 123-149. 


\section{Layered circle packings}

[6] J. W. S. Rayleigh, On the theory of resonance, Collected Scientific Papers, vol. 1, Cambridge University Press, Cambridge, 1899, pp. 33-75.

[7] B. Rodin and D. Sullivan, The convergence of circle packings to the Riemann mapping, J. Differential Geom. 26 (1987), no. 2, 349-360.

[8] R. Siders, Layered circlepackings and the type problem, Proc. Amer. Math. Soc. 126 (1998), no. 10, 3071-3074.

[9] W. Thurston, The finite Riemann mapping theorem, International Symposium at Purdue University on the Occasion of the Proof of the Bieberbach Conjecture, Purdue University, West Lafayette, USA, March 1985.

[10] , The Geometry and Topology of 3-Manifolds, Princeton University Notes, preprint, 2002.

David Dennis: Department of Mathematics and Statistics, Texas Tech University, Lubbock, TX 79409-1042, USA

E-mail address: david.dennis@ttu.edu

G. Brock Williams: Department of Mathematics and Statistics, Texas Tech University, Lubbock, TX 79409-1042, USA

E-mail address: williams@math.ttu.edu 


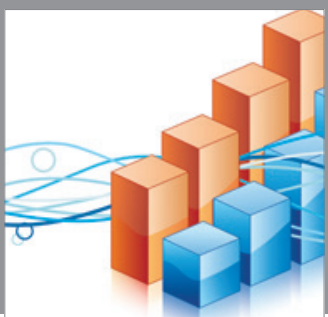

Advances in

Operations Research

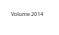

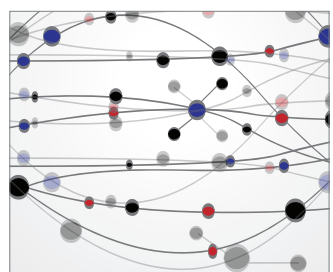

\section{The Scientific} World Journal
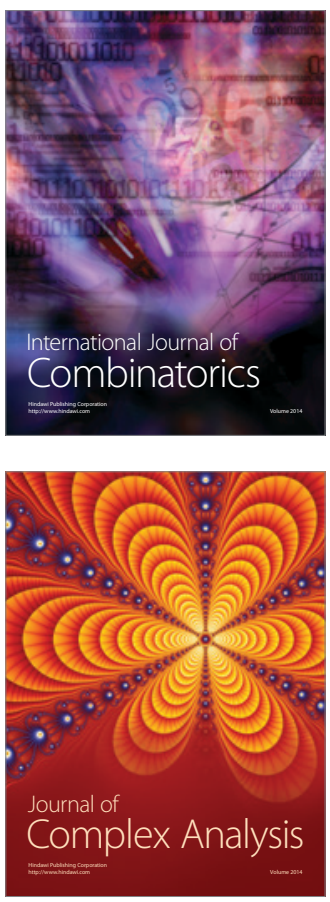

International Journal of

Mathematics and

Mathematical

Sciences
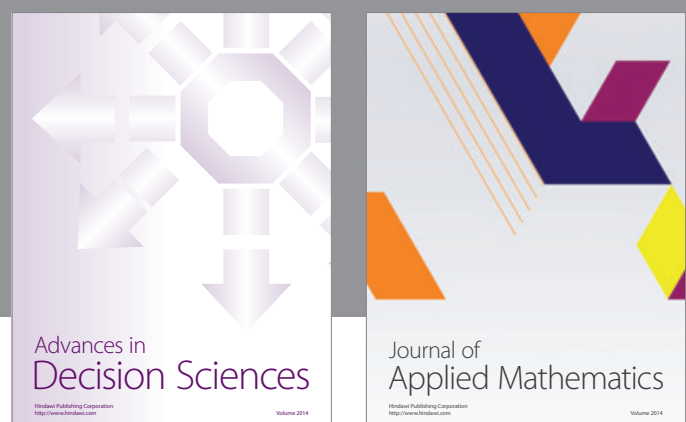

Journal of

Applied Mathematics
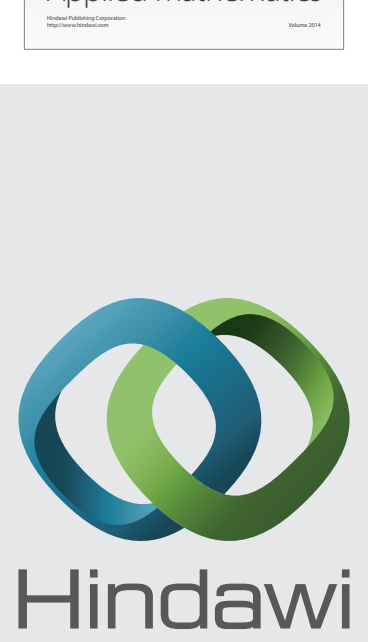

Submit your manuscripts at http://www.hindawi.com
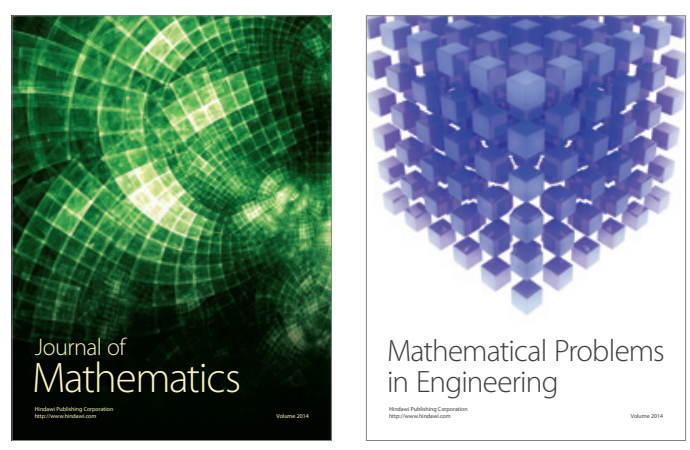

Mathematical Problems in Engineering
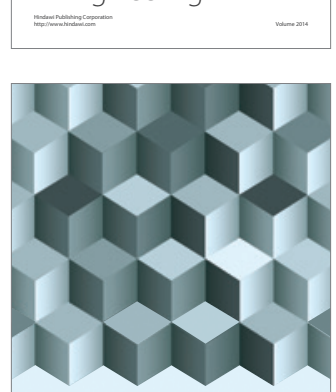

Journal of

Function Spaces
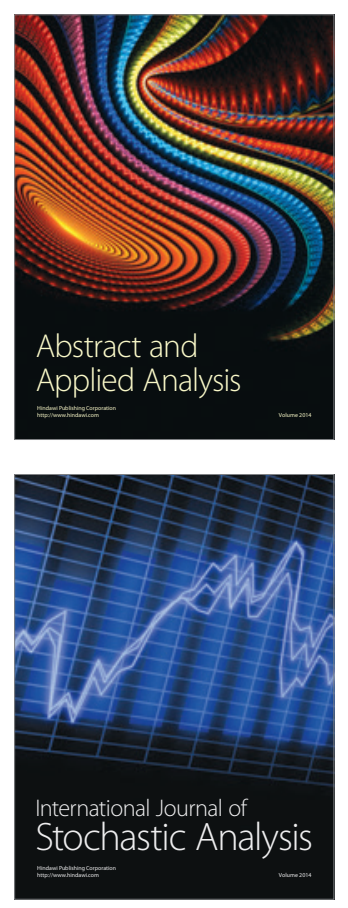

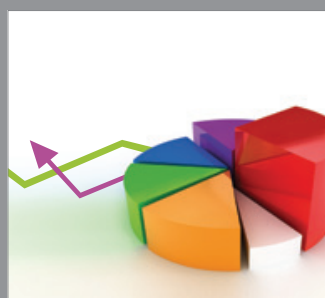

ournal of

Probability and Statistics

Promensencen
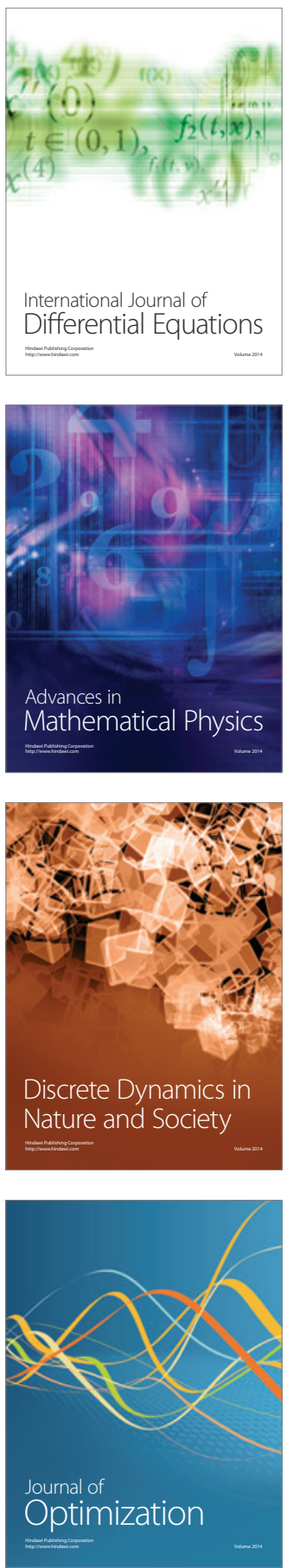